\title{
Pore-polymer interaction reveals nonuniversality in forced polymer translocation
}

\author{
V. V. Lehtola, K. Kaski, and R. P. Linna \\ Department of Biomedical Engineering and Computational Science, Aalto University, P.O. Box 12200, FI-00076 Aalto, Finland
}

(Received 10 July 2010; revised manuscript received 21 August 2010; published 17 September 2010)

\begin{abstract}
We present a numerical study of forced polymer translocation by using two separate pore models. Both of them have been extensively used in previous forced translocation studies. We show that variations in the pore model affect the forced translocation characteristics significantly in the biologically relevant range of the pore force, i.e., the driving force. Details of the model are shown to change even the obtained scaling relations, which is a strong indication of strongly out-of-equilibrium dynamics in the computational studies which have not yet succeeded in addressing the characteristics of the forced translocation for biopolymers at realistic length scale.
\end{abstract}

DOI: 10.1103/PhysRevE.82.031908

PACS number(s): 87.15.A-, 82.35.Lr

\section{INTRODUCTION}

Polymer translocation has been under intensive research for the past decade due to its relevance for, e.g., ultrafast DNA sequencing [1-5] and many biological processes [6]. Most of the computational research on forced translocation, where the polymer is driven through a nanoscale pore by a potential, has dealt with fairly weak pore potential or force and assumed close-to-equilibrium dynamics. Recently, hydrodynamics has been shown to significantly affect forced translocation for experimentally and biologically relevant force magnitudes $[7,8]$. The pore geometry has been noted to have effect on the experimental translocation process (see, e.g., [5]). In addition, the effect of the pore model has been discussed in $[9,10]$. However, the significance of the pore model in the forced polymer translocation has not yet been determined.

We have recently made a comparison of the unforced and forced translocations [11]. In the first case, the process was seen to be close to equilibrium, as expected, and accordingly its dynamics turned out to be robust against variations in the computational model. However, in the second case the observed out-of-equilibrium characteristics for biologically and experimentally relevant pore force magnitudes made the dynamics more sensitive to differences in the computational models [8,12]. Accordingly, we expect the pore model to play a significant role in the forced translocation case [11].

So far, we have consistently used a cylindrical pore implemented by a potential symmetrical around the pore axis $[8,11,12]$. However, this differs from the typical pore implementation where the pore is formed by removing one particle from a wall consisting a monolayer of immobile pointlike particles [13-17].

A notable difference is that the latter pore implementation results in a driving potential that is inhomogeneous in the direction of the pore axis. In the case of lattice Boltzmann simulations $[18,19]$ these bead pore models include both the square- $[16,17]$ and cylindrically [20] shaped pores. Typically, the pore-polymer interaction is solely repulsive, but the effect of attractive interaction has been investigated in a twodimensional model [21].

In order to investigate how susceptible translocation dynamics is to variations in the pore model we have imple- mented in our computational translocation model also the bead pore used in [14]. We compare translocation processes for the bead pore and our original cylindrical pore. The model dependency is expected to be strongest in the pore region under strong forcing. We also try to evaluate the effect of the pore model for moderate forcing.

When the force bias inside the pore is small, the velocities involved are small enough for the hydrodynamics to be neglected [8,11], and the Langevin dynamics is a valid choice for the computational model. Assuming that the polymer remains close to equilibrium throughout the translocation, the control parameter may be defined as the ratio of the total external force and the solvent friction, $\zeta=f_{\text {tot }} / \xi$ as done by Luo et al. in [14]. Then the translocation dynamics would depend only on $\zeta$. They reported this to be the case even for a long polymer chain driven through the pore with a significant value of total pore force $f_{\text {tot }}$. The scaling of the average translocation time with respect to the chain length, $\tau \sim N^{\beta}$, would then be determined solely by $\zeta$. This is in clear contradiction with our earlier observation that the scaling exponent $\beta$ increases with the force due to crowding of the monomers on the trans side $[8,12]$. In addition, the effect of the pore model along with other model-dependent factors would in this case be negligible [11].

The increase in $\beta$ with force cannot hold asymptotically as pointed out in [14] since ultimately very long polymers would translocate faster with a smaller pore force. From Fig. 1 (b) in [8] it can be evaluated that this unphysical condition would be seen for $N \gtrsim 10^{6}$ for the pore force magnitudes of 1 and 10. This is comparable to actual DNA lengths of $10^{6}, \ldots, 10^{9}$ that are far beyond polymer lengths of $N$ $\lesssim 1000$ used in simulations. The translocation dynamics then would have to be different for $N>10^{6}$, which is in keeping with our earlier qualitative description of the forced translocation $[8,12]$. The polymers were seen to be driven out of equilibrium throughout the translocation already for modest pore force magnitudes. The continually increasing drag force on the cis and crowding on the trans side were seen to change the force-balance condition, which is directly reflected on the translocation dynamics. This force balance is sure to be different for very long polymers.

The suggested control parameter $\zeta$, if applicable, would imply that the forced translocation could be completely characterized through varying it, which again is in stark contrast 


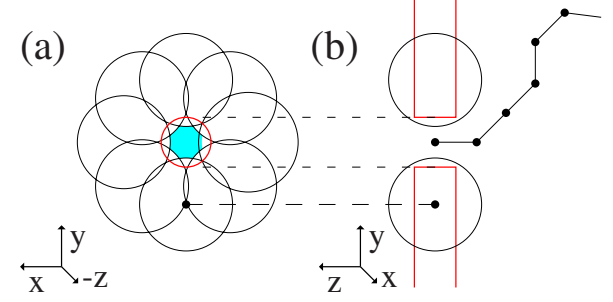

FIG. 1. (Color online) Schematic depiction of the two pore models. (a) The pores are viewed from the trans side along the $z$ axis. The small red circle depicts the cylindrical pore of diameter $1.2 \sigma$. The bead pore is defined by the eight beads each at distance $1.5 \sigma$ from the $z$ axis. The pore beads are drawn with circles using the LJ potential cutoff length $2^{1 / 6}$ as their radius. The light blue area in the center of the pore indicates the region where polymer beads have no interaction with the pore beads. In contrast, the cylinder pore model has a damped-spring-like potential that acts on particles everywhere inside the pore. (b) Side view. The polymer about to translocate $(s=1)$ is drawn as connected dots. The potentials of the two pore models differ in both the $x y$ plane and along the $z$ axis.

to our observation that the forced translocation is a nonuniversal out-of-equilibrium process for biologically relevant magnitudes of pore force $[8,12]$. Therefore, it is essential to determine if this contradiction arises from using different pore models.

This paper is organized such that we first describe the polymer model in Sec. II and the translocation models in Sec. III. The relation between computational and physical parameters is discussed in Sec. IV. The results are presented and discussed in Sec. V. We conclude the paper by summarizing our findings in Sec. VI.

\section{POLYMER MODEL}

The standard bead-spring chain is used as a coarsegrained polymer model with the Langevin dynamics. In this model adjacent monomers are connected with anharmonic springs, described by the finitely extensible nonlinear elastic potential,

$$
U_{F E N E}=-\frac{K}{2} R^{2} \ln \left(1-\frac{r^{2}}{R^{2}}\right),
$$

where $r$ is the length of an effective bond and $R=1.5$ is the maximum bond length. The Lennard-Jones $(\mathrm{LJ})$ potential,

$$
\begin{gathered}
U_{L J}=4 \epsilon\left[\left(\frac{\sigma}{r}\right)^{12}-\left(\frac{\sigma}{r}\right)^{6}\right], \quad r \leq 2^{1 / 6} \sigma, \\
U_{L J}=0, \quad r>2^{1 / 6} \sigma,
\end{gathered}
$$

is used between all beads of distance $r$ apart. The parameter values were chosen as $\epsilon=1.2, \sigma=1.0$, and $K=60 / \sigma^{2}$. The used LJ potential mimics good solvent.

\section{TRANSLOCATION MODELS}

To perform simulations of polymer translocation in three dimensions we use our translocation model based on Lange- vin dynamics [11]. Hence, the time derivative of the momentum of bead $i$ reads as

$$
\dot{\mathbf{p}}_{i}(t)=-\xi \mathbf{p}_{i}(t)+\eta_{i}(t)+f\left(\mathbf{r}_{i}\right),
$$

where $\xi, \mathbf{p}_{i}(t), \eta_{i}(t)$, and $f\left(\mathbf{r}_{i}\right)$ are the friction constant, the momentum, random force of the bead $i$, and the external driving force, respectively. $f\left(\mathbf{r}_{i}\right)$ is constant but exerted only inside the pore. For unforced translocation, $f\left(\mathbf{r}_{i}\right)=0$. $\xi$ and $\eta_{i}(t)$ are related by the fluctuation-dissipation theorem. The dynamics was implemented by using velocity Verlet algorithm [22].

In our model the wall containing the pore was implemented as a surface on which no-slip boundary conditions are applied to the polymer beads. The two different computational pore models to be compared can be described as follows (see Fig. 1):

Cylindrical pore. The pore, aligned with the $z$ axis, is of diameter $1.2 \sigma(=1.2 b)$ and length $l=n b$, where $b=1$ is the Kuhn length of the model polymer, and $n$ is either 1 or 3 . The pore is implemented by a cylindrically symmetric damped harmonic potential that pulls the beads toward the pore axis passing through the middle of the pore in the $z$ direction. The pore is $n b$ long, so the total pore force is taken as $f_{\text {tot }}=n f$, where $f$ is the external driving force applied on each polymer bead inside the pore.

Bead pore. An octagonal composition of eight immobile particles was used as the bead pore model, as depicted in Fig. 1. This pore model includes a region where a polymer bead can reside without interacting with the pore beads (see Fig. 1 ). The effective pore length is approximately $b$, so $f_{t o t}=f$. Unlike in our model, in [14] also the wall was made of immobile particles. We verified that this has no effect on the translocation dynamics by reproducing essentially identical results on $\tau$ for the force magnitudes that were reported in [14].

In our previous Langevin dynamics simulations with the cylindrical pore $[8,11]$, parameter values $\xi=0.73, m=16$, and $k T=1$ were used for the friction constant, the polymer bead mass, and the temperature in reduced units, respectively. Hence, for long times the one-particle self-diffusion constant was obtained from Einstein's relation as $D_{0}=k T / \xi m$ $\approx 0.086$. Time steps of 0.01 and 0.03 were (previously) used in the forced and unforced simulations, respectively. In this paper, to compare the two pore models, we have used $m=1$, and $k T=1.2$ as in [14], unless otherwise noted. Here, the time step is typically 0.001 .

The number of beads $N$ is odd for polymers initially placed halfway inside the pore and even for polymers having initially only the first bead(s) inside the pore (see Fig. 1). Before allowing translocation a polymer is let to relax for longer than its Rouse relaxation time. We register events when a segment $s=s_{0}$ in the pore is replaced with the segment $s_{0}-1$ or $s_{0}+1$. The polymer is considered translocated, when it has completely exited the pore to the trans side. Exit to either trans or cis side is regarded as an escape from the pore. 


\section{RELATING THE COMPUTATIONAL AND THE PHYSICAL PORE FORCE}

The external driving force, called the total pore force, $f_{\text {tot }}=n f$, where $n$ is the number of polymer beads inside the pore and $f$ is the $z$-directional driving force exerted on each bead inside the pore, will be taken as the effective pore force in our model. The thermal energy is set to $k T=1.2$ in reduced units and the length scale is set by the polymer bond length $b$. The magnitude of the effective pore force is then determined with respect to thermal fluctuations by comparing $f_{\text {tot }} b$ with $k T$. It is noteworthy, however, that since the pore length is on the order of the polymer bond length, there exists an inevitable computational artifact due to the discrete polymer model. The external force $f$ is exerted on beads residing in the pore, whose number $n$ does not remain constant during translocation. In addition this number is different for different pore lengths, so the pore force does not increase strictly linearly with the pore length. These effects are, however, mitigated by the fact that $n$ averaged over translocation time is constant.

Correspondence between computational and physical length scale can be established by taking the polymer bond length $b$ as the Kuhn length for the physical polymer. In SI units the bond length for our freely jointed chain model polymer can be obtained as $\tilde{b}=2 \lambda_{p}$, where $\lambda_{p}$ is the persistence length, $40 \AA$ for a single-stranded DNA (ssDNA) [23]. The total pore force in SI units, $\widetilde{f}_{\text {tot }}$, is then obtained from the dimensionless total pore force $f$ by relating $\tilde{f}_{\text {tot }} \tilde{b} / k_{B} \widetilde{T}$ $=f_{\text {tot }} b / \mathrm{kT}$. Here, $k_{B}$ is the Boltzmann constant, and the physical temperature $\widetilde{T}$ is taken to be $300 \mathrm{~K}$. Thus, the effective pore force of $f_{\text {tot }}=1$ corresponds to $\tilde{f}_{\text {tot }}=1.2 \mathrm{pN}$ for a ssDNA. To relate to experiments, in the $\alpha$-HL pore a typical pore potential of $\approx 120 \mathrm{mV}$ would correspond to $\tilde{f}_{\text {tot }}$ $\approx 5 \mathrm{pN}$ when Manning condensation leading to drastic charge reduction is taken into account $[24,25]$. The used computational pore force magnitudes are thus in the physically relevant range [8].

\section{RESULTS}

\section{A. Forward transfer probability}

Previously [11], we have shown that during the unforced translocation the polymer remains close to equilibrium, so that the forward transition probabilities,

$$
P_{f}(s) \propto\left(1-\frac{1}{s}+\frac{1}{N-s}\right)^{1-\gamma},
$$

derived from the free energy apply in three dimensions, where $\gamma=0.69$. Including the pore force in the calculation only introduces a prefactor to the above $P_{f}(s)$ not changing its form, i.e., dependence on $s$. For this to hold, the translocating polymer has to remain close to equilibrium. Indeed, except for a small increase due to the force term, the shapes of the measured $P_{f}(s>N / 2)$ for the unforced and forced translocations with the total pore force $f_{\text {tot }}=0.1$ are seen to be similar (cf. Fig. 2). $P_{f}$ 's for the two pore models do not differ

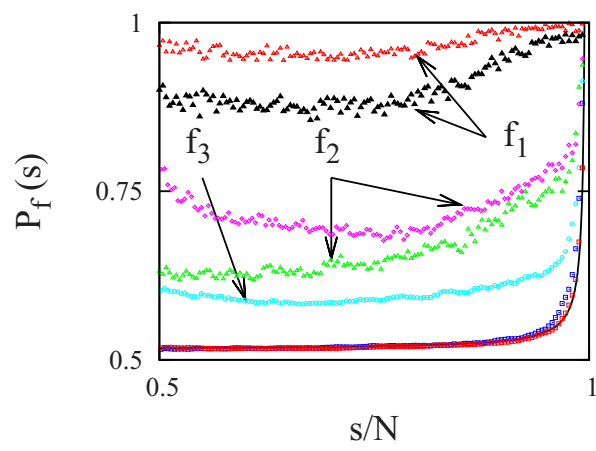

FIG. 2. (Color online) Forward transfer probabilities $P_{f}$ as functions of the reaction coordinate normalized with polymer length, $s / N$. The data are given for the bead (bd) and cylindrical (cyl) pores. Here, $\xi=0.7$ and $N$ is 255 or 256 , depending on the polymer's initial position. The pore force $f_{\text {tot }}$ has the following values from top to bottom: $f_{1}=5.0$ (cyl,bd), $f_{2}=1.17$ (bd), $f_{3}=0.5$ (bd). At the bottom are $P_{f}$ for $f_{\text {tot }}=0.1$ for the bead (bd, distinct $\square$ from the solid curve) and the cylindrical (cyl) pore (red) obtained from simulations together with the black solid curve calculated from Eq. (4) for the unforced case. For the $f_{\text {tot }}$ values of $0.1,0.5$, and $1.17\left(f_{2}\right.$ upper curve) the polymer was initially placed halfway through the pore $s=(N-1) / 2$. For the $f_{\text {tot }}$ values of 5.0 (both) and $1.17\left(f_{2}\right.$ lower curve), the polymer started from the cis side $s=1$. The shape of the probability curve depends on the pore model $\left(f_{1}\right)$, polymer's initial position $\left(f_{2}\right)$, and changes with the force.

appreciably for this small pore force. Hence, we conclude that the transition probabilities can be obtained from equilibrium framework for pore force magnitudes on the order of 0.1 .

For the bead pore already for the force $f_{\text {tot }}=0.5$, the $P_{f}(s)$ curve differs from the equilibrium shape, as shown in Fig. 2. Hence, the effect of the pore force can no more be taken as a small perturbation to equilibrium dynamics. In keeping with this, the transition probabilities $P_{f}(s>N / 2)$ for $f_{\text {tot }}=1.17$ are seen to depend on the polymer's initial position, here either on the cis side or halfway through the pore. In other words, the transition probability from the current state $s$ to the next depends on the path through which this state was arrived at. The polymer cannot then have relaxed to equilibrium between previous transitions.

Using the cylindrical pore, the probability $P_{f}$ is seen to be almost constant and close to 1 for all $s$ with $f_{\text {tot }}=5$ (see Fig. 2 ). This is in accordance with our previous results that the polymer was seen to almost always translocate with $f_{\text {tot }} \simeq 3$, which means that also in this case $P_{f}(s>0)$ would be close to $1[8]$. However, $P_{f}(s)$ for the bead pore deviates significantly from $P_{f}(s)$ for the cylinder pore when $f_{\text {tot }}=5$ (see Fig. 2 ). Thus, it is evident that the pore force magnitudes in the two model pores do not have an exact correspondence for large pore force magnitudes.

The pore force at which translocation is seen to be a strongly out-of-equilibrium process is surprisingly low for both pore models. It may be compared with the random force in the one-particle Langevin equation satisfying fluctuationdissipation theorem in three dimensions, for which $\left\langle f(t) f\left(t^{\prime}\right)\right\rangle=6 k T \xi \delta\left(t-t^{\prime}\right)$. With the parameter values $\xi=0.7$ and $k T=1.2$ used in our simulations, this yields $f \simeq 2.2$. 

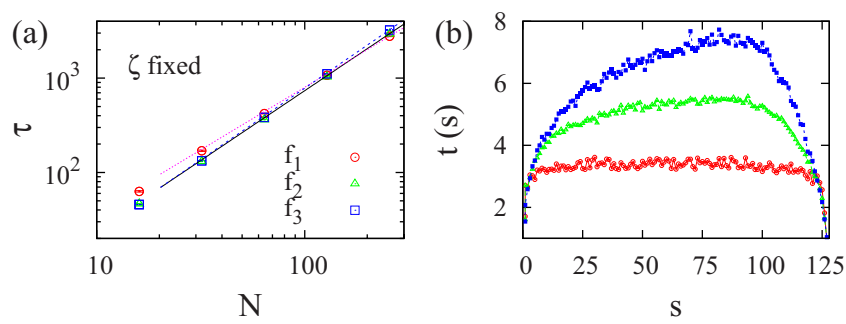

FIG. 3. (Color online) Results from 3D Langevin dynamics with a bead pore model. Keeping $\zeta=f_{\text {tot }} / \xi$ fixed, results for three pairs of parameter values $\left(f_{t o t}, \xi\right):(1.17,0.7),(5,3)$, and $(10,6)$, are shown. $f_{\text {tot }}=f_{1}, f_{2}$, and $f_{3}$, respectively. (a) Scaling of the average translocation time $\tau$ with respect to the chain length $N, \tau \sim N^{\beta}$. The scaling exponent $\beta$ has values of $1.32 \pm 0.03$ (dotted magenta line), $1.48 \pm 0.03$ (solid black line), and $1.52 \pm 0.03$ (dashed blue line) for the parameter pairs, respectively. The exponents are from fits for $N \in[32,64,128,256]$. (b) Average transition times $t(s)=t(s-1$ $\rightarrow s$ ) for $N=128$. Plots from bottom to top are for $f_{\text {tot }}=f_{1}, f_{2}$, and $f_{3}$, respectively. The transition time profile changes significantly when increasing the pore force even though the proposed control parameter $\zeta$ is kept fixed.

Already at fairly moderate pore force values, a local force balance governs the translocation dynamics. This was first proposed by Sakaue [26] and demonstrated in simulations by us $[8,12]$. Considering a concept of "mobile beads" near the pore, we obtained and described qualitatively the scaling relation $\tau \sim N^{1+\nu-\sigma} / f_{\text {tot }}$, where the parameter $\sigma$ taking into account the varying number of mobile beads diminishes toward zero with increasing pore force $[8,12]$. Sakaue recently gave a fairly quantitative out-of-equilibrium formulation for the scaling $\tau \sim N^{1+\nu} / f_{\text {tot }}[27]$. However, neither description takes into account the crowding of monomers on the trans side shown to be significant for these pore force magnitudes [8].

Here, we present results from simulations of the polymer translocation covering the relevant range of the pore force. When $f_{\text {tot }} b / k T \lesssim 0.1, P_{f}(s)$ attains the equilibrium shape, and when $f_{\text {tot }} b / k T \underset{\gtrsim}{\approx}, P_{f}(s)$ is close to 1 .

\section{B. Proposed control parameter}

Using the bead pore, we first measured the scaling exponent $\beta$ for different values of pore force $f_{\text {tot }}$ while keeping the proposed control parameter $\zeta=f_{\text {tot }} / \xi$ fixed. For the parameter pair $\left(f_{\text {tot }}, \xi\right)$, values $(1.17,0.7),(5,3)$, and $(10,6)$ were used [see Fig. 3(a)]. Similar average translocation times $\tau$ were obtained for large pore force magnitudes, $f_{\text {tot }}=\{5,10\}$. However, $\tau$ differs appreciably for $f_{\text {tot }}=1.17$, resulting in a different value for the scaling exponent $\beta$.

The measured average waiting (or state transition) times $t(s)=t(s-1 \rightarrow s)$ are compared in Fig. 3(b). The shown three plots for $t(s)$ are for the above-mentioned values of $\left(f_{t o t}, \xi\right)$. In the limit $f_{\text {tot }} b / k T \ll 1$, the average state transition times $t(s)$ were seen to be similar. This is in accord with the transition times in the unforced translocation obeying a close-toPoissonian distribution [11]. When $f_{t o t} b / k T \gg 1$ the friction of the solvent dominates the stochastic term in the Langevin equation [Eq. (3)]. The transition time profiles for the bead pore are qualitatively similar with those obtained for the cylindrical pore with different parameters [12].
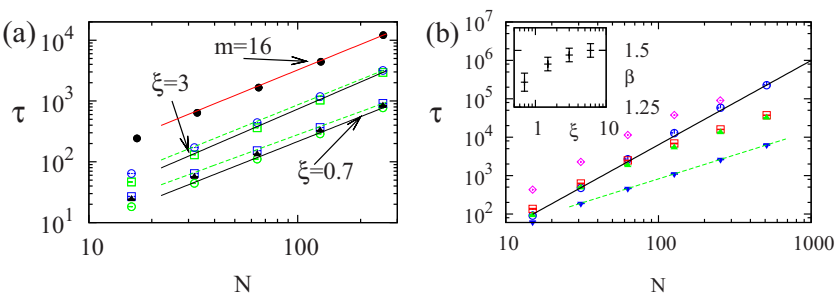

FIG. 4. (Color online) Scaling of the average translocation time with respect to the chain length, $\tau \sim N^{\beta}$. The results are from 3D Langevin dynamics simulations with both cylindrical (cyl) and bead (bd) pore models with different pore lengths $l=n b$. (a) The constant total pore force $f_{t o t}=5$. The exponents are from fits for $N$ $\in[32,64,128,256]$. For $\xi=0.7$, the scaling exponent $\beta$ $=1.26 \pm 0.02$ (blue $\square), 1.27 \pm 0.02(\mathbf{\Delta})$, and $1.36 \pm 0.03$ (green $\bigcirc$ ), for $l=3$ (cyl), $l=1$ (cyl), and $l=1$ (bd), respectively. For $\xi=3$, the scaling exponent $\beta=1.39 \pm 0.02$ (blue $\bigcirc$ ) and $1.48 \pm 0.02$ (green $\square$ ), for $l=3$ (cyl) and $l=1$ (bd), respectively. Increasing the bead mass from 1 to 16 increases the absolute value of $\tau$, and $\beta$ $=1.40 \pm 0.03(-)$ for $\xi=0.7$ (cyl). See text for details. (b) For $f_{\text {tot }}$ $=0.5, \xi=0.7(\boldsymbol{\nabla}:$ bd $)$ the polymers are initially halfway through the pore. We obtain $\beta=1.25 \pm 0.04$ (green dashed line) with $N$ $\in\{31,63,127,255,511\}$. For $f_{\text {tot }}=0.1, \xi=0.7$ (red $\square$ : cyl, $\mathbf{\Delta}:$ bd) polymers are also initially halfway through the pore. The absolute values of $\tau$ differ slightly for the two pore models with shorter polymer chains but not so for $N \simeq 511$ when all chains escape to the trans side. For a very low force $f_{\text {tot }}=0.01$ (blue $\bigcirc$ : bd) even the longest $(N=511)$ chains may escape to the cis side. We obtain $\beta$ $=2.2 \pm 0.1$ (solid black line). For reference, results with $f_{\text {tot }}=0.1$, $\xi=6(\diamond: \mathrm{bd})$ are shown. Inset: the scaling exponent $\beta$ as a function of the friction constant $\xi, f_{\text {tot }}=5$ (bd). For $\xi=\{0.7,1.5,3.0,6.0\}$, we have $\beta=1.36 \pm 0.04,1.44 \pm 0.03,1.48 \pm 0.03$, and $1.50 \pm 0.03$, respectively.

From the different scaling of the translocation times obtained for large and small pore force magnitudes and the different average state transition times, we conclude that $\zeta$ which was kept fixed cannot be a universal control parameter for forced translocation regardless of the pore model. This is in keeping with our previous finding that the forced translocation is a highly out-of-equilibrium nonuniversal process for the biologically and experimentally relevant pore force range $[8,12]$.

\section{Pore model matters in forced translocation}

Originally, the need to compare the cylindrical and bead pore models arose from the differences in the scaling of the translocation time $\tau$ with the polymer length $N$ in forced translocation obtained using these two pore models. The translocation times averaged over at least 1000 and at most 2500 runs are shown in Fig. 4(a) as functions of $N$. The total pore force is constant, $f_{\text {tot }}=5$, and chosen to be within the experimentally relevant range [8]. For the friction parameter $\xi$ three values, $0.7,3$, and 6 , are used. For the two separate pore models we find clear differences in both the absolute value of $\tau$ and its apparent scaling. Regardless of the value for $\xi$, we obtain a smaller scaling exponent $\beta$ for the cylindrical than for the bead pore. In addition, increasing $\xi$ from 0.7 to 6 increases $\beta$ for both pore models [see the inset in 
Fig. 4(b)]. This in part explains the different results obtained with the two pores as the effective friction experienced by a chain is different inside them. This difference is naturally enhanced at higher velocities.

Our previous results were obtained with the polymer bead mass $m=16$ due to the requirement of the stochastic rotation dynamics that the polymer beads should be heavier than solvent beads $[8,12]$. Therefore, we checked how the bead mass affects the characteristics of the translocation time. Increasing the bead mass from 1 to 16 is seen not only to increase the average translocation time $\tau$ but also to change its apparent scaling with polymer length $\tau \sim N^{\beta}$ [see Fig. 4(a)]. To be certain, we checked this using two independent Langevin algorithms.

In accordance with our previous findings for cylindrical pore, $\beta$ was seen to increase with pore force also for the bead pore. For $f_{\text {tot }}=\{0.5,1.17,5,10\}$, the scaling exponents $\beta$ $=1.25 \pm 0.04$ [Fig. 4(b)], $\beta=1.32 \pm 0.03$ [Fig. 3(a)], $\beta$ $=1.36 \pm 0.03$ [Fig. 4(a)], and $\beta=1.37 \pm 0.03$ (not shown) were obtained, respectively. Surprisingly, for the cylindrical pore $\beta$, which increased substantially with increasing $f_{\text {tot }}$ when $m=16[8,12]$, did not show such a strong tendency when $m=1$. Changing $m$ seems then to change even the qualitative characteristics of forced polymer translocation.

The change in $\beta$ in the scaling $\tau \sim N^{\beta}$ due to a change in $\xi$ is hardly surprising. However, $\beta$ changing with $m$ is a bit more subtle. It suggests that we are actually looking at the forced translocation in a continually changing or transient stage. This is in accord with our previous finding that for polymers of lengths that are used in simulations, $N \leq 1000$, the number of moving polymer beads changes throughout the translocation, and thus continually alters the forcebalance condition resulting from the forced translocation taking place out of equilibrium [8]. Also the crowding of the polymer beads on the trans side, whose relaxation toward equilibrium is slower than the translocation rate, changes the force balance continually. Hence, the simulated forced translocation processes do not reveal the asymptotic $\left(N>10^{6}\right)$ characteristics for experimentally relevant pore force magnitudes. Then reporting scaling exponents for such a forced translocation seems unwarranted. The remaining question of interest then is why does the forced translocation exhibit the scaling albeit with varying exponents?

\section{Translocation at low pore force}

For simulations at low pore force the chains were initially placed halfway through the pore unlike at large pore force where the polymer was placed, so that only its end was inside the pore. At the very low force of $f_{\text {tot }}=0.1$ the pore model was found to affect only slightly the absolute value of $\tau$ [see Fig. 4(b)]. For the bead pore model chains of length $N \leq 127$ had a finite probability to escape to the cis side. Unlike for the bead pore, even some of the 255 beads long polymers escaped to the cis side for the cylindrical pore. This is probably due to the cylindrical pore aligning the polymer chain toward the pore axis in the middle, thus effectively reducing the friction between the polymer and the pore.

For the pore force $f_{\text {tot }}=0.01$ we obtain $\beta=2.2 \pm 0.1$ [see Fig. 4(b)], which is the expected exponent for unforced translocation, $\beta=2 \nu+1=2.2$, since $\nu=0.6$ has been measured for the swelling exponent in our model [12]. Approaching the unforced case makes the translocation dynamics increasingly robust to variations in the model, which is a natural consequence of the small pore force not dominating entropic forces. We regard the pore force magnitude of 0.1 already small enough for the translocating polymer to remain close to equilibrium (see Fig. 2). However, we did not obtain a clear scaling for $f_{\text {tot }}=0.1$ for either pore models, but $\beta$ close to the unforced translocation value $2 \nu+1$ was obtained for chains shorter than $N=127$, while a lower value of $\beta$ was obtained for longer chains. This applies for both $\xi=0.7$ and $\xi=6$ [see Fig. 4(b)]. We expect the lower value of $\beta$ to be $1+\nu$ (see [28]), although a wider range of $N$ would be needed to confirm this. This apparent crossover behavior for low pore force is outside the scope of the present paper and calls for a separate more thorough investigation.

For the presumably small pore force $f_{\text {tot }}=0.5$ we obtained $\beta=1.25 \pm 0.04$. This differs considerably from $\beta$ $=1.58 \pm 0.03$ reported in [14]. From Fig. 2 it can be seen that $P_{f}$ for $f_{t o t}=0.5$ clearly deviates from the close-to-unforced form of $P_{f}$ for $f_{\text {tot }}=0.1$. Together with the low value of $\beta$ this observation suggests that $f_{\text {tot }}=0.5$ is large enough to drive the polymer more and more out of equilibrium as the translocation proceeds. The different values for $\beta$ here and in [14] also support this view since the two simulations started from different initial positions. When the relaxation time of the polymer toward thermal equilibrium is slower than the translocation time, the process possesses memory or correlation over time, i.e., the memory function $M\left(t-t_{0}\right) \neq \delta\left(t-t_{0}\right)$. Thus, a polymer starting halfway through the pore is in a different state than a polymer that has arrived at this position and started from another initial position.

The data presented for different pore force magnitudes here and in Sec. V C show that for $f_{\text {tot }} \geq 0.5$ the value of $\beta$ increases with increasing pore force $[8,12]$ also for the bead pore. For very low pore force $\beta$ is found independent of the pore force.

\section{SUMMARY}

In summary, we have simulated forced polymer translocation in three dimensions by using Langevin dynamics. We have implemented two pore models in our algorithm: (i) a pore surrounded by eight immobile beads, which we call bead pore, and (ii) a cylindrical pore where a damped harmonic potential confines the beads inside the pore region. The present study compares the effects these two pores have on the forced translocation.

We measured the forward transition probabilities $P_{f}(s)$ to determine the range of pore force that would be sufficiently large to include cases where polymer translocation takes place close to and strongly out of equilibrium. We found that the polymer remains close to equilibrium when the total pore force $f_{t o t} b / k T \lesssim 0.1$. Here, the translocation dynamics was found to be robust to variations in the translocation model, such as the details of the pore. $P_{f}(s)$ was found to deviate significantly from the close-to-equilibrium form already for a pore force as small as 0.5 . The polymer was found to be 
driven far from equilibrium when $f_{\text {tot }} b / k T \gtrsim 5$.

For small pore force magnitudes the forced translocation processes are identical for the two pore models. However, the translocation characteristics were found to be increasingly model dependent when the pore force is increased. This is a natural consequence of the dynamics of the forced translocation being determined by a continually changing forcebalance condition when the pore force is large enough, i.e., $\left(f_{t o t} b / k T \gtrsim 0.5\right)$. Accordingly, it seems that universal exponents for the forced translocation cannot be found in the biologically relevant pore force regime. In addition, attempts to define a control parameter whose magnitude would consistently determine these exponents would seem futile, which we showed for one proposed candidate by using the bead pore. Qualitatively the forced translocation exhibited similar characteristics with both pore models, most notably the increase with the pore force of the exponent determining the relation between the average translocation time and the polymer length. In our view this is another indication of the continually changing force-balance condition governing the highly nonequilibrium forced translocation process.

\section{ACKNOWLEDGMENTS}

One of the authors (V.V.L.) thanks Dr. K. Luo for email exchanges. This work was supported by the Academy of Finland (Project No. 127766). The computational resources of CSC-IT Centre for Science, Finland are acknowledged.
[1] J. J. Kasianowicz, E. Brandin, D. Branton, and D. W. Deamer, Proc. Natl. Acad. Sci. U.S.A. 93, 13770 (1996).

[2] W. Storm et al., Nano Lett. 5, 1193 (2005).

[3] J. Li, M. Gershow, D. Stein, E. Brandin, and J. A. Golovchenko, Nature Mater. 2, 611 (2003).

[4] A. Meller, L. Nivon, and D. Branton, Phys. Rev. Lett. 86, 3435 (2001).

[5] M. Zwolak and M. Di Ventra, Rev. Mod. Phys. 80, 141 (2008).

[6] B. Alberts et al., Molecular Biology of the Cell (Garland Publishing, New York, 1994).

[7] M. Fyta, S. Melchionna, S. Succi, and E. Kaxiras, Phys. Rev. E 78, 036704 (2008).

[8] V. V. Lehtola, R. P. Linna, and K. Kaski, EPL 85, 58006 (2009).

[9] D. K. Lubensky and D. R. Nelson, Biophys. J. 77, 1824 (1999).

[10] E. Slonkina and A. B. Kolomeisky, J. Chem. Phys. 118, 7112 (2003).

[11] V. V. Lehtola, R. P. Linna, and K. Kaski, Phys. Rev. E 81, 031803 (2010).

[12] V. V. Lehtola, R. P. Linna, and K. Kaski, Phys. Rev. E 78, 061803 (2008).

[13] K. Luo, S. T. T. Ollila, I. Huopaniemi, T. Ala-Nissila, P. Pomorski, M. Karttunen, S.-C. Ying, and A. Bhattacharya, Phys. Rev. E 78, 050901(R) (2008).

[14] K. Luo, T. Ala-Nissila, S.-C. Ying, and R. Metzler, EPL 88,
68006 (2009).

[15] M. G. Gauthier and G. W. Slater, Eur. Phys. J. E 25, 17 (2008).

[16] M. Fyta, E. Kaxiras, S. Melchionna, and S. Succi, Comput. Sci. Eng. 10(4), 10 (2008).

[17] M. Fyta, S. Melchionna, E. Kaxiras, and S. Succi, Multiscale Model. Simul. 5, 1156 (2006).

[18] R. Benzi, S. Succi, and M. Vergassola, Phys. Rep. 222, 145 (1992).

[19] S. Chen and G. D. Doolen, Annu. Rev. Fluid Mech. 30, 329 (1998).

[20] M. Bernaschi, S. Melchionna, S. Succi, M. Fyta, and E. Kaxiras, Nano Lett. 8, 1115 (2008).

[21] K. Luo, T. Ala-Nissila, S.-C. Ying, and A. Bhattacharya, Phys. Rev. Lett. 99, 148102 (2007).

[22] W. van Gunsteren and H. Berendsen, Mol. Phys. 34, 1311 (1977).

[23] B. Tinland, A. Pluen, J. Sturm, and G. Weill, Macromolecules 30, 5763 (1997).

[24] A. Meller, J. Phys.: Condens. Matter 15, R581 (2003).

[25] A. F. Sauer-Budge, J. A. Nyamwanda, D. K. Lubensky, and D. Branton, Phys. Rev. Lett. 90, 238101 (2003).

[26] T. Sakaue, Phys. Rev. E 76, 021803 (2007).

[27] T. Sakaue, Phys. Rev. E 81, 041808 (2010).

[28] Y. Kantor and M. Kardar, Phys. Rev. E 69, 021806 (2004). 